\title{
IL-37 Was Involved in Progress of Acute Myeloid Leukemia Through Regulating IL-6 Expression
}

\author{
Xiaonan Wei ${ }^{1, *}$ \\ Yulan $\mathrm{Li}^{2}$,* \\ Guili Zhang ${ }^{2}$ \\ $\mathrm{Na} \mathrm{Wang}^{2}$ \\ Miaomiao $\mathrm{Mi}^{3}$ \\ Yu Xin ${ }^{4}$ \\ Huihui Jiang ${ }^{3}$ \\ Chengming Sun $\left(\mathbb{D}^{2}\right.$ \\ 'Qingdao Women and Children's \\ Hospital, Qingdao University, Qingdao, \\ Shandong, 266000, People's Republic of \\ China; ${ }^{2}$ Center for Laboratory Diagnosis, \\ Yantai Yuhuangding Hospital Affiliated to \\ Qingdao University, Yantai Shandong, \\ 264000, People's Republic of China; \\ ${ }^{3}$ School of Medicine, Qingdao University, \\ Qingdao, Shandong, 266000, People's \\ Republic of China; ${ }^{4}$ School of Clinical \\ Medical, Binzhou Medical University \\ Clinical Laboratory, Yantai Yuhuangding \\ Hospital, Yantai, Shandong, 264000, \\ People's Republic of China \\ *These authors contributed equally to \\ this work
}

Correspondence: Chengming Sun Email chengmingsun@163.com
Background: Interleukin-37, which was discovered in 2000, is a natural suppressor of immune and inflammatory responses. Recent studies reported that IL-37 was abnormally expressed in several tumor patients, including those with hepatocellular carcinoma, gastric cancer, lung cancer, colon cancer, epithelial ovarian cancer, and multiple myeloma. However, the expression and potential function of IL-37 in leukemia remain unknown.

Objective: The aim of this study was to evaluate IL-37 as a prognostic factor and its possible mechanism of action.

Methods: Polymerase chain reaction products were analyzed by agarose gel electrophoresis and were purified and subsequently sequenced by a genetic testing laboratory. Human PBMC was purified from whole blood samples by using Ficoll-Paque PLUS. The concentrations of human IL-37 and human IL-6 were measured using enzyme-linked immunosorbent assay (ELISA) kits. Results: IL-37, especially isoform $b$ and $d$, was expressed in the bone marrow of AML, CML, ALL, and CLL. Importantly, IL-37 expression was downregulated in newly diagnosed AML patients and restored in patients in complete remission. Moreover, a significant association was found between IL-37 expression and NPM1 mutation or possible prognosis evaluated by karyotype and gene mutation. Further analysis revealed that IL-37 expression was negatively correlated with IL-6 expression. With regard to the mechanism, recombinant human IL-37 could suppress IL-6 expression stimulated by LPS in PBMC of AML patients. Conclusion: Our study suggested that IL-37 may be an important prognostic factor in AML and is involved in AML via the IL-6 signaling pathway, indicating that IL-37 is an innovative research strategy for AML pathogenesis and therapy.

Keywords: interleukin-37, acute myeloid leukemia, IL-6, prognosis

\section{Introduction}

Acute myeloid leukemia (AML) is a malignant hematologic neoplasm that is characterized by aberrant proliferation and bone marrow infiltration of myeloid progenitor cells (leukemic blasts). ${ }^{1}$ AML is highly heterogeneous with respect to cytomorphology, immunophenotype, cytogenetics, and gene mutation, as well as response to therapy. ${ }^{2}$ It is the most common acute leukemia and often occurs in older persons ( $>65$ years old). ${ }^{1}$ However, more than $50 \%$ of patients will experience relapse due to the large heterogeneity, and the cure rate is relatively low $(<15 \%))^{3,4}$ This somatic heterogeneity poses great challenges to AML treatment. Therefore, exploring unifying mechanisms in the initiation and progression of AML is necessary, providing guidance for more effective treatment options.

IL-37, a newly discovered member of the IL-1 family, has been identified as a natural suppressor of innate immunity and inflammatory responses. ${ }^{5-8}$ Five different 
splice variants of IL-37 exist, which are referred to as IL$37 \mathrm{a}-\mathrm{e} .{ }^{9,10} \mathrm{IL}-37 \mathrm{~b}$ is a biologically functional isoform with the greatest amounts of exons and detected in various human tissues, including brain lymph nodes, thymus, bone marrow, lung, thymus, testis, placenta, uterus, immune cells, and tumors. ${ }^{7,11,12}$ IL-37 could suppress the expression of TLRinduced pro-inflammatory cytokines in PBMCs and human monocytic cell line THP-1 such as IL-1 $\beta$, IL-6, and TNF$\alpha{ }^{7,12}$ IL-37 protects against several inflammatory models in IL-37 transgenic mice, such as colitis, ${ }^{13}$ endotoxic shock, ${ }^{7}$ and obesity-induced inflammation. ${ }^{14}$ Administration of rhIL37 attenuated multiple diseases such as rheumatoid arthritis, ${ }^{15}$ systemic lupus erythematosus, ${ }^{16}$ Con A-induced hepatitis, ${ }^{17}$ and psoriasis. ${ }^{18}$ However, the biological role of IL-37 in tumor remains to be further investigated. Recent studies reported that IL-37 mediated antitumor activity in several solid tumors. Our previous study found that in lung adenocarcinoma, IL-37 inhibited tumor metastasis via inhibiting Rac1 activation. ${ }^{19}$ Wang et al reported that IL-37 inhibited the proliferation and invasion of human cervical cancer cells through STAT3 signaling. ${ }^{20}$ Zhao et al found that the tumor-suppressive role of IL-37 in the growth of human hepatocellular carcinoma (HCC) was exerted through recruiting CD57+NK cells. ${ }^{21}$ However, whether IL-37 is involved in the progression of AML remains unknown.

A recent study reported that the serum levels of IL-37 were low and negatively associated with angiogenesis in multiple myeloma, a bone marrow-derived tumor. ${ }^{22}$ AML is another hematological malignancy. The activity of general immune system is modulated by leukemia cells in AML. The dysregulated cytokine expression promoted hematological malignancies and also showed significant association with resistance and relapse of AML. ${ }^{23-25}$ All the above-mentioned evidence prompts us to suppose whether IL-37 is involved in AML by regulating the expression of pro-inflammatory cytokines.

\section{Materials and Methods}

\section{Patients and Samples}

This study recruited 117 patients with leukemia and 15 patients with IDA at the Affiliated Yantai Yuhuangding Hospital of Qingdao University from September 2018 to December 2019. Of these 117 patients, 108 were AML. The number of patients with CML, ALL, and CLL was 3, respectively. All patients were diagnosed through cytomorphology, cytogenetic, and molecular genetic analysis of BM aspirates, and they were classified based on the revised 2008
World Health Organization (WHO) criteria. Sixty-six healthy individuals who were receiving a health checkup served as the control group. The serum supernatants were obtained from the peripheral blood after being centrifuged at $3000 \mathrm{rpm}$ for $10 \mathrm{~min}$ at $4^{\circ} \mathrm{C}$ and stored at $80^{\circ} \mathrm{C}$ for the next analysis. Bone marrow (BM) aspirate specimens of 14 patients with newly diagnosed AML, 4 AML patients achieving complete remission, 4 relapsed or refractory AML patients, and 15 IDA patients were collected. The characteristics of patients with newly diagnosed AML are summarized in Table 1. This study was approved by the Ethics Committee of the Affiliated Yantai Yuhuangding Hospital of Qingdao University (2017210) and conducted in accordance with the Declaration of Helsinki. All participants provided signed informed consent.

\section{RNA Isolation, PCR, and Sequencing}

Total RNA from bone marrow was isolated using TRIzol reagent (Invitrogen, Carlsbad, CA, USA), and complementary DNA was obtained using a reverse transcription kit (TransGen Biotech, Beijing, China). For semiquantitative $\mathrm{PCR}$, polymerase chain reaction (PCR) products were analyzed by agarose gel electrophoresis and were purified and sequenced subsequently by a genetic testing laboratory ${ }^{26}$ (Beijing Liuhe Huada Gene Technology Co., Ltd., Beijing, China). Quantitative RT-PCR was performed using a SYBR RT-PCR kit (TransGen Biotech, Beijing, China) and 7500 Fast Real-time PCR system ${ }^{27,28}$ (Applied Biosystems, Foster City, CA, USA). The PCR products were verified by melting curve analysis. The relative messenger RNA (mRNA) levels of target genes were calculated using the $2^{-\Delta \Delta \mathrm{Ct}}$ method. ${ }^{29}$ Primers for human IL-37, IL-37a, IL-37b, IL-37c, IL-37d, IL-37e, IL-6, IL-1 $\beta$, $\mathrm{TNF} \alpha$, and $\beta$-actin are shown in Table 2.

\section{PBMC Isolated, Culture with Human Recombinant IL-37}

Human PBMC was purified from whole blood samples by using Ficoll-Paque PLUS ${ }^{30,31}$ (GE Healthcare. Illinois, USA) and seeded into 12-well plates with $1 \mathrm{ml}$ RPMI 1640 containing $10 \%$ FBS. After being cultured overnight, the cells were pretreated with gradient concentrations of recombinant IL-37b from $0 \mathrm{pg} / \mathrm{mL}$ to $10 \mathrm{ng} / \mathrm{mL}$ for $12 \mathrm{~h}$. After lipopolysaccharide (LPS) stimulation for $8 \mathrm{~h}$, the supernatants were collected by centrifugation at $1000 \mathrm{~g}$ for $5 \mathrm{~min}$ and were used for direct analysis or stored at $-80^{\circ} \mathrm{C}$. 
Table I Relationship Between the Expression of IL-37 and Clinical Features of Acute Myeloid Leukemia

\begin{tabular}{|c|c|c|c|}
\hline Patient's Parameter & Low $(n=33)$ & High $(n=18)$ & $P$-value \\
\hline Sex, male/female & $17 / 16$ & 9/9 & 0.918 \\
\hline Median age, years (range) & $57(6-88)$ & $65(28-80)$ & 0.561 \\
\hline Age, $<60 / \geqq 60$ & $18 / 15$ & $7 / 11$ & 0.285 \\
\hline Median WBC, $\times 10^{9} / \mathrm{L}$ (range) & $5.68(10.49-201.99)$ & $29.32(1.10-124.85)$ & 0.053 \\
\hline Median hemoglobin, g/L (range) & $71(37-126)$ & 71 (34-99) & 0.370 \\
\hline Median platelets, $\times 10^{9} / \mathrm{L}$ (range) & $54(9-5 \mid 4)$ & $45(11-169)$ & 0.561 \\
\hline BM blasts, \% (range) & $55.5(20.5-94.5)$ & $68.5(24-93.5)$ & 0.827 \\
\hline PM blasts, \% (range) & $44(0-96)$ & $44(0-97)$ & 0.391 \\
\hline \multicolumn{4}{|l|}{ Karyotype classification } \\
\hline Favorable & $7(21 \%)$ & $5(28 \%)$ & 0.423 \\
\hline Intermediate & $15(46 \%)$ & 7 (39\%) & \\
\hline Poor & $10(30 \%)$ & $2(11 \%)$ & \\
\hline No data & I (3\%) & $4(22 \%)$ & \\
\hline \multicolumn{4}{|l|}{ Karyotype } \\
\hline Normal & II (34\%) & $8(44 \%)$ & 0.117 \\
\hline $\mathrm{t}(8,2 \mathrm{I})$ & I (3\%) & $2(12 \%)$ & \\
\hline$t(16,16)$ & $0(0 \%)$ & I (5\%) & \\
\hline $\mathrm{t}(15,17)$ & $3(9 \%)$ & $0(0 \%)$ & \\
\hline+8 & $3(9 \%)$ & I (5\%) & \\
\hline Others & $7(2 \mid \%)$ & $0(0 \%)$ & \\
\hline Complex & 7 (2।\%) & $3(17 \%)$ & \\
\hline No data & I (3\%) & $3(17 \%)$ & \\
\hline \multicolumn{4}{|l|}{ Gene mutation } \\
\hline \multicolumn{4}{|l|}{ CEBPA } \\
\hline Wide type & 22 (67\%) & $13(72 \%)$ & 0.502 \\
\hline Mutated & $2(6 \%)$ & $2(11 \%)$ & \\
\hline No data & $9(27 \%)$ & $3(17 \%)$ & \\
\hline \multicolumn{4}{|l|}{ NPMI } \\
\hline Wide type & $22(67 \%)$ & $9(5 \%)$ & $0.025^{*}$ \\
\hline Mutated & $2(6 \%)$ & $6(33 \%)$ & \\
\hline No data & 9 (27\%) & $3(17 \%)$ & \\
\hline \multicolumn{4}{|l|}{ FLT3 } \\
\hline Wide type & $20(61 \%)$ & II (61\%) & 0.360 \\
\hline Mutated & $4(12 \%)$ & $4(22 \%)$ & \\
\hline No data & 9 (27\%) & $3(17 \%)$ & \\
\hline \multicolumn{4}{|l|}{ c-KIT } \\
\hline Wide type & 24 (73\%) & $13(17 \%)$ & 0.142 \\
\hline Mutated & $0(0 \%)$ & $2(11 \%)$ & \\
\hline No data & 9 (27\%) & $3(17 \%)$ & \\
\hline
\end{tabular}

Note: *Compare with Mutated group, the difference was statistically significant $\mathrm{P}<0.05$.

\section{Cytokine Concentration Measurement}

The concentrations of human IL-37 and human IL- 6 were measured using enzyme-linked immunosorbent assay (ELISA) kits (R\&D System, Minneapolis, USA) according to the manufacturer's instruction. IL-37 and IL-6 levels were calculated according to the standard curves.

\section{Statistical Analysis}

All statistical analyses were performed using SPSS version 22.0 (SPSS Inc., Chicago, IL, USA) and GraphPad Prism software (La Jolla, CA, USA). Data are shown as mean \pm standard deviation. Differences between two groups were analyzed using Student's $t$-test. Chi-square test was used to 
Table 2 Primer Sequences for Specific Genes

\begin{tabular}{|l|c|}
\hline Primer Name & Primer Sequence \\
\hline IL-37 sense & 5'-TTCTTTGCATTAGCCTCATCCTT-3' \\
IL-37 antisense & 5'-CGTGCTGATTCCTTTTGGGC-3' \\
IL-37a sense & 5'-GCGCTTAAGAGG TCCAAAGGT-3' \\
IL-37a antisense & 5'-GCTATGAGATTCCCAGAGTCCAG-3' \\
IL-37b sense & 5'-TCACACAAGTCCAAAGGTGA-3' \\
IL-37b antisense & 5'- AGCCAGCTTCATCAGTTTCT-3' \\
IL-37c sense & 5'-TCACACAAAGATCTTCTTTGCA-3' \\
IL-37c antisense & 5'-CAGCCAGCTTCATCAGTTTC-3' \\
IL-37d sense & 5'-GCTTAGAAGGTCCAAAGGTGAA-3' \\
IL-37d antisense & 5'-GAGCTCAAGGATGAGGCTAATG-3' \\
IL-37e sense & 5'- GCTGCTTAGAAGAGATCTTCTTT-3' \\
IL-37e antisense & 5'-CTGAAGGGATGGATGACTTTG-3' \\
IL-6 sense & 5'-GCCAGAGCTGTGCAGATGAG-3' \\
IL-6 antisense & 5'-TCAGCAGGCTGGCATTTG-3' \\
IL-I $\beta$ sense & 5'-GCAATGAGGATGACTTGTTCTTTG-3' \\
IL-I $\beta$ antisense & 5'-CAGAGGTCCAGGTCCTGGAA-3' \\
TNF $\alpha$ sense & 5'-TGCTTGTTCCTCAGCCTCTT-3' \\
TNF $\alpha$ antisense & 5'-CAGAGGGCTGATTAGAGAGAGGT-3' \\
$\beta$-actin sense & 5'-GTCATTCCAAATATGAGATGCGT-3' \\
$\beta$-actin antisense & 5'-GCTATCACCTCCCCTGTGTG-3' \\
\hline
\end{tabular}

analyze the relationship between IL-37 levels and the clinicopathological characteristics. The correlation between IL37 and IL- 6 was assessed by Spearman correlation analysis. A $P$-value $<0.05$ was considered significant.

\section{Results}

\section{IL-37 Expression in the Bone Marrow of Leukemia Patients}

A previous study reported that IL-37 could be expressed in the bone marrow of healthy people. ${ }^{32}$ We first analyzed the expression of IL-37 in the bone marrow of four different types of leukemia by using RT-PCR. IL-37 was observed in the bone marrow of AML, CML, ALL, and CLL (Figure 1A). Further sequencing analysis of PCRamplified products confirmed the result (Figure 1B). Among all the five IL-37 isoforms, IL-37a, b, and d were predicted to be functional, ${ }^{33}$ and we investigated the expression of five different isoforms by using isoformspecific primers. As shown in Figure 1C, the transcripts for IL-37b and $d$ could be detected in the bone marrow of leukemia patients, but the transcripts for IL-37a, c, and e were absent. The data suggest that the IL-37 isoforms detected in the bone marrow of leukemia patients are biologically functional and may participate in the development of leukemia.

\section{IL-37 Expression Was Decreased and Significantly Associated with NPMI Mutation in AML Patients}

AML is the most common acute leukemia and patients often have a poor prognosis. Thus, we further explored IL-37 mRNA $(n=14)$ and protein levels $(n=51)$ in newly diagnosed AML patients. The reduction in the IL37 mRNA level was observed in the bone marrow but was statistically non-significant (Figure 2A and B). This result may be due to the short half-life of the cytokine mRNA because of the presence of an mRNA instability motif in the coding sequence of exon $5 .{ }^{34}$ Importantly, IL-37 expression was significantly downregulated in the serum of newly diagnosed AML patients compared with that in healthy controls (Figure 2C). To further analyze the relationship between IL-37 expression and clinicopathological features, we divided the newly diagnosed AML patients into IL-37 high-expression and IL-37 low-expression groups. A significant association was found between IL-37 expression and NPM1 mutation status (Table 2). These results indicate that the abnormal expression of IL-37 may be an important factor in the microenvironment of AML.

\section{Low IL-37 Expression Predicted Poor Prognosis of AML}

To further investigate the possible role of IL-37 in the progress of AML, we next detected IL-37 levels in patients with newly diagnosed AML, complete remission (CR) AML, and relapsed or refractory $(R / R)$ AML. The decreased IL-37 levels in newly diagnosed AML was reversed in CR AML. However, IL-37 expression was still kept at a low level in patients with $R / R$ AML (Figure 3A-C). Moreover, a significant difference was observed in IL-37 expression between favorable and intermediate/poor prognosis evaluated by both karyotype and gene mutation (Figure 4). These results indicate that IL-37 expression may be associated with the prognosis of AML patients.

\section{IL-37 Expression Was Negatively Correlated with IL-6 Expression in the Serum of AML Patients}

Recent studies indicate that abnormal cytokine signaling pathways may significantly contribute to the growth, resistance, and relapse of AML. ${ }^{35}$ Therefore, the levels 
A

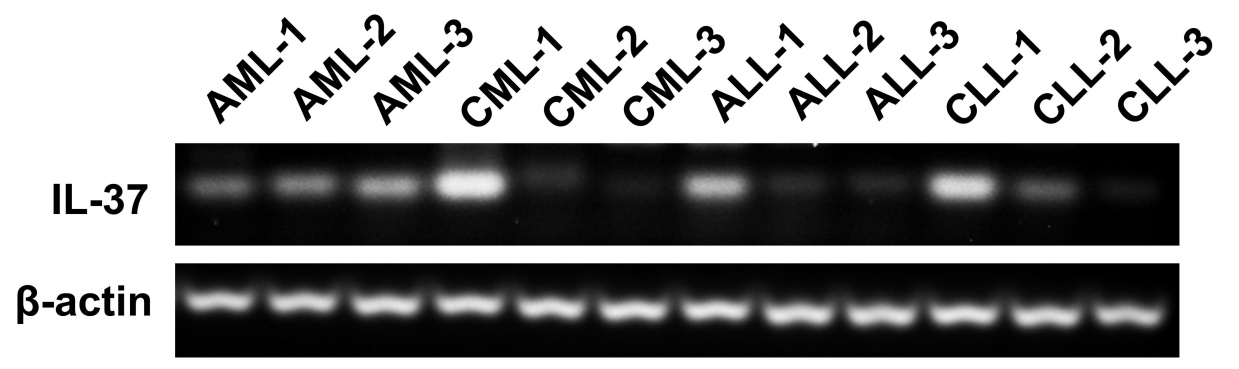

B

IL-37

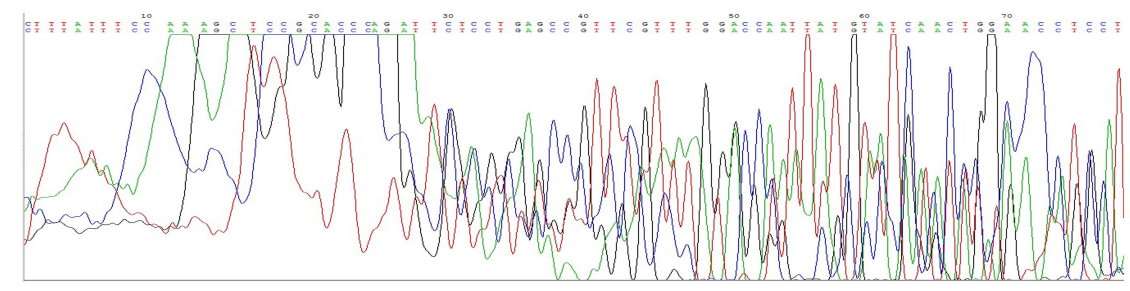

Sequencing

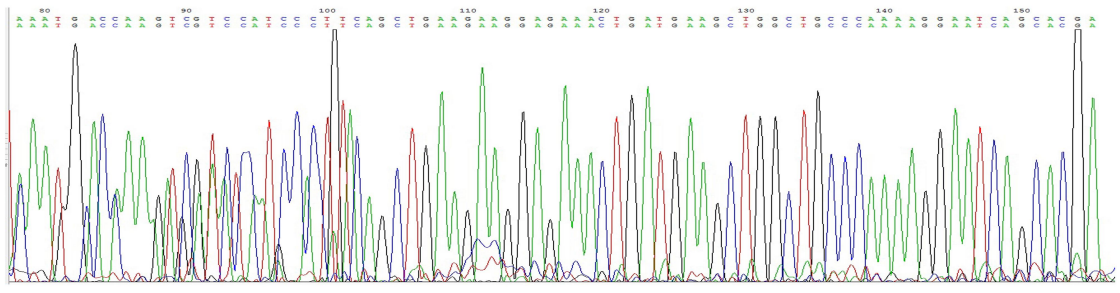

C

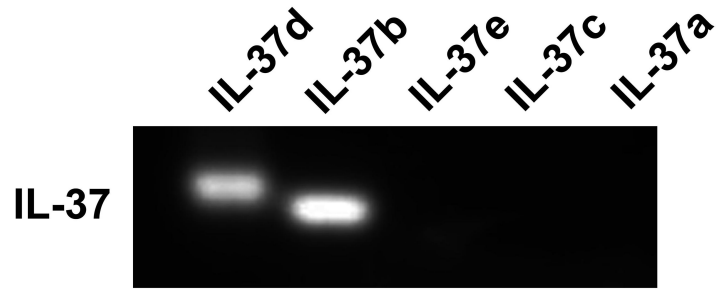

Figure I IL-37 mRNA levels in bone marrow of patients with leukemia. (A) IL-37 mRNA levels in patients with different types of leukemia by semiquantitative PCR. (B) Sanger sequencing for PCR products of IL-37. (C) mRNA levels of five IL-37 isoforms.

of cytokines that may modulate AML development, such as IL-1 $\beta$, IL-6, and TNF- $\alpha$, were measured by using RT-PCR in bone marrow and ELISA in the serum. The mRNA levels of IL-1 $\beta$ and TNF- $\alpha$ increased, but no statistical significance was observed (Figure 5A and B). However, IL-6 mRNA and protein levels were significantly upregulated in newly diagnosed AML compared with the healthy controls (Figure 5C and D). The upregulation in IL-6 protein levels was reversed in $\mathrm{CR} A M L$ and reoccurred in $\mathrm{R} / \mathrm{R}$ AML (Figure 5D). Further correlation analysis revealed that IL-37 expression was negatively correlated with IL-6 expression in the serum of AML patients (Figure 5E). These results indicate that IL-37 may be involved in the development of AML via regulating IL-6 expression.

\section{IL-37 Suppressed IL-6 Expression in PBMCs of AML Patients}

To demonstrate the effect of IL-37 on IL-6 expression of AML patients, PBMCs were extracted in peripheral blood and stimulated by LPS and recombinant human IL-37 (rhIL-37) protein. Morphological changes of PBMCs were observed after stimulation by LPS alone for 12 hours, such as changes in cell shape and formation of pseudopodia, thereby indicating that PBMCs were activated. However, compared with the group stimulated with LPS alone, the group pretreated with rhIL-37 did not exhibit obvious morphological changes (Figure 6A). Importantly, the pretreatment with rhIL-37 at $1 \mathrm{ng} / \mathrm{mL}$ in the supernatant of PBMCs could weaken the increase in IL-6 expression induced by LPS (Figure 6B). These results 
A

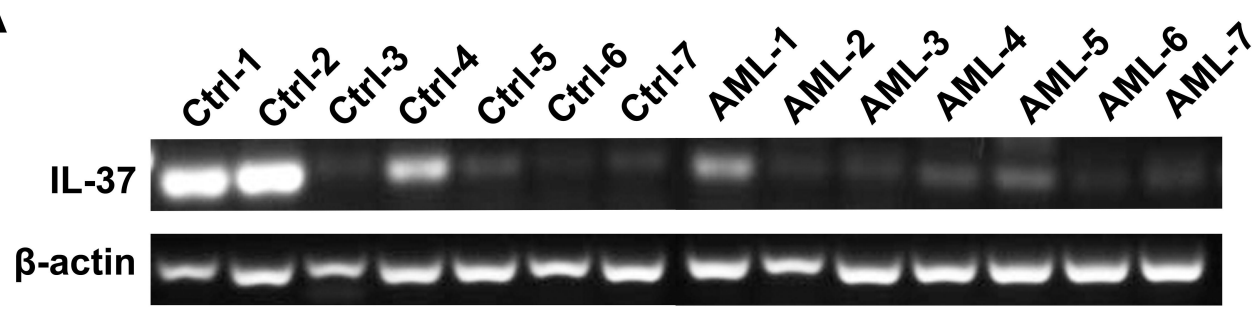

B

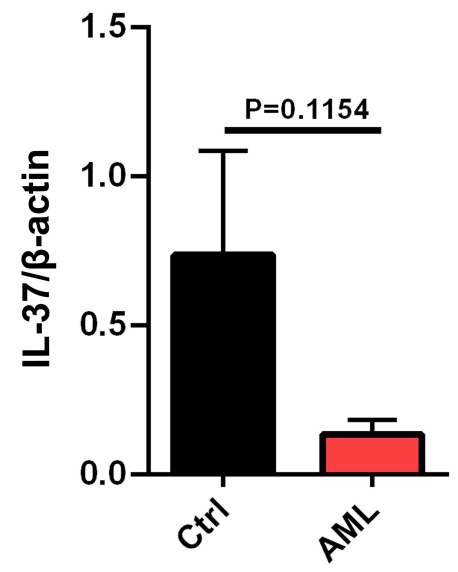

C

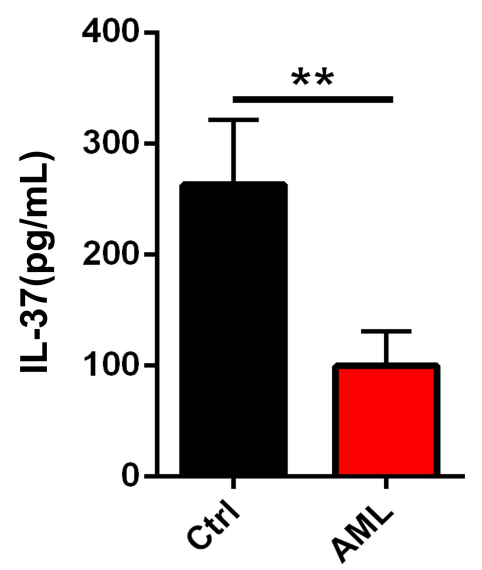

Figure 2 IL-37 levels were decreased in patients with newly diagnosed AML. (A and B) IL-37 mRNA levels in patients with newly diagnostic AML and IDA controls by semiquantitative PCR. (C) Serum IL-37 levels in patients with newly diagnosed AML $(n=36)$ and healthy controls $(n=55)$ by ELISA. Data show the mean \pm SD. **P $<0.0$ I.

A

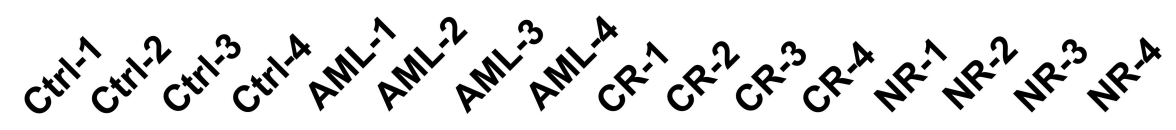

IL-37

$\beta$-actin
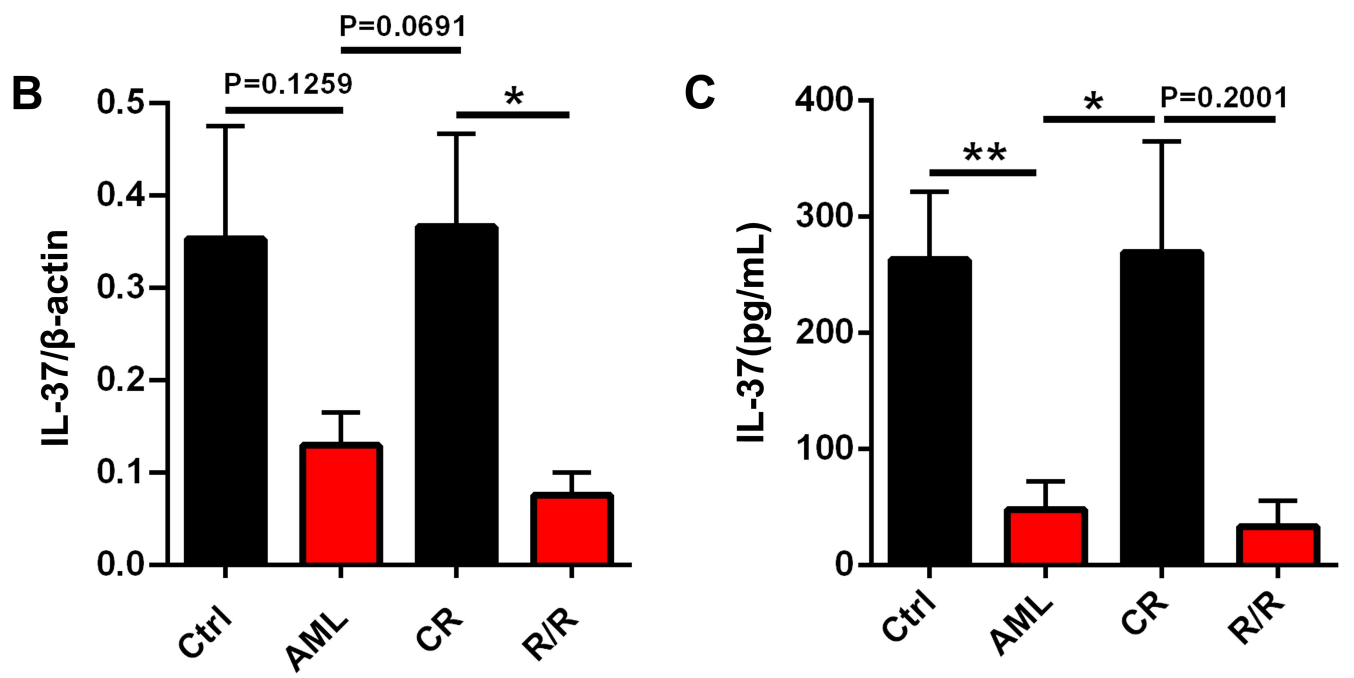

Figure 3 IL-37 mRNA and serum levels in patients with newly diagnosed AML, CR AML and R/R AML. (A and B) IL-37 mRNA levels in patients with newly diagnosed AML, CR AML, R/R AML and IDA controls by semiquantitative PCR. (C) Serum IL-37 levels in patients with newly diagnosed $A M L(n=36), C R$ AML $(n=43), R / R$ AML ( $=12)$ and healthy controls $(n=55)$ by ELISA. Data show the mean $\pm S D$. $*^{*} p<0.01$, ${ }^{*} p<0.05$. 


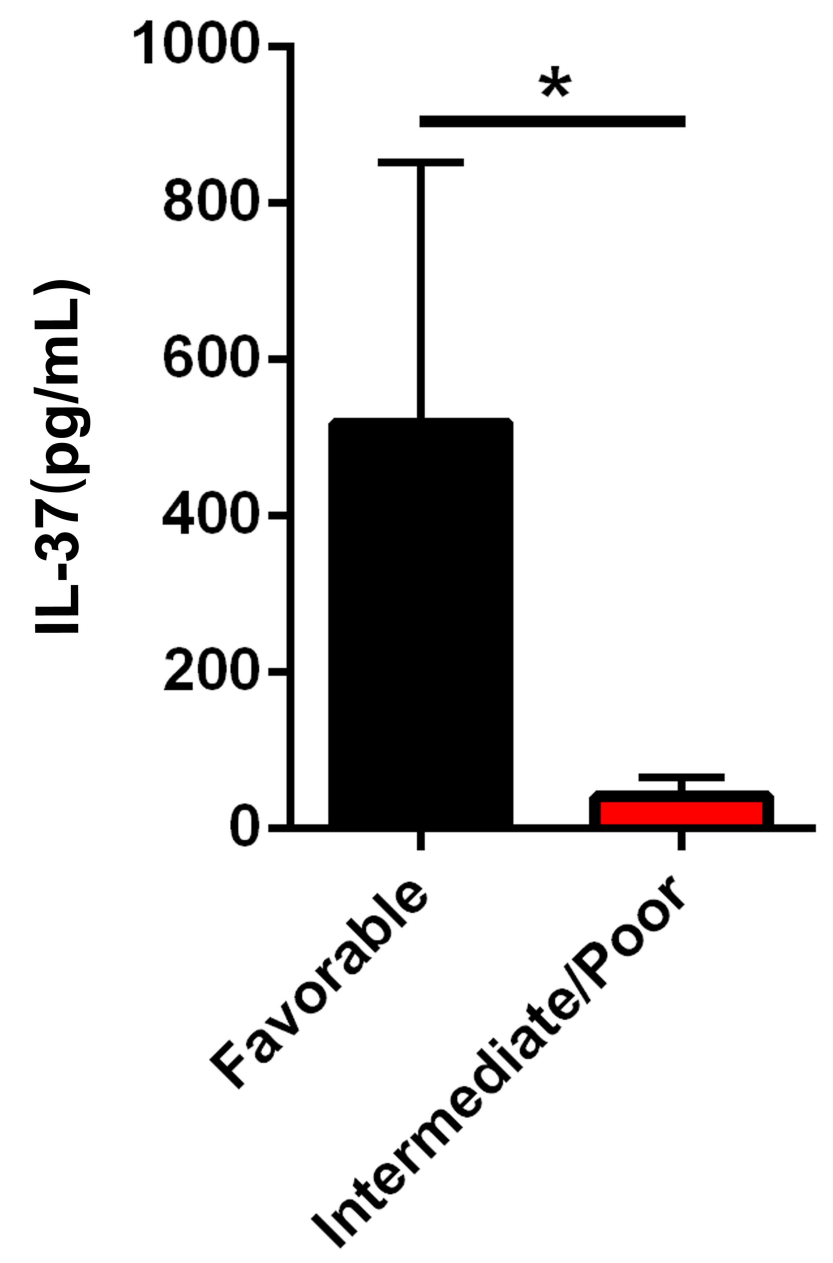

Figure 4 Serum IL-37 levels in patients with favorable prognosis $(n=8)$ and intermediate/poor prognosis $(n=23)$ evaluated by karyotype and gene mutation. Data show the mean $\pm S D$. $*_{p}<0.05$

indicate that IL-6 could be inhibited by IL-37 in PBMCs of AML patients.

\section{Discussion}

IL-37 is a newly identified member of the IL-1 family. Recent studies demonstrate that IL-37 may have an important tumor suppressive role in tumors, which has five isoforms, and the expression of different isoforms is tissue specific. $^{32}$ IL-37 could be detected in many human and tumor tissues. However, the IL-37 levels in bone marrow of leukemia are still unknown. IL-37 occurred in four different types of leukemia, namely, AML, CML, ALL, and CLL (Figure 1A). Furthermore, IL-37b and IL-37d were the main isoforms expressed in the bone marrow of AML, while IL-37a, IL-37c, and IL-37e were not detected (Figure 1C). The five isoforms of IL-37 can all be expressed in healthy bone marrow, and IL-37d and e are present in the bone marrow only. ${ }^{9,36}$ This finding is different from our results, indicating that AML may change the expression ratio of isoforms in bone marrow. However, the above hypothesis needs to be further confirmed.

Of the five isoforms, IL-37b is the only one that is demonstrated to have biological functions. Our recent study first reported that IL-37d was another functional isoform and participated in the inflammation in a Smad3dependent way. ${ }^{37}$ This evidence indicates that IL-37 expressed in the bone marrow of AML is biologically functional and may be involved in the development of AML. The findings of this study demonstrate that IL-37 was markedly downregulated in newly diagnosed AML (Figure 2) and restored in CR AML. However, IL-37 expression was suppressed again in patients with $R / R$ AML (Figure 3). Further analysis revealed that IL-37 expression was significantly associated with the possible prognosis of AML patients evaluated by both karyotype and gene mutation (Figure 4). The somatic heterogeneity, including cytomorphology, immunophenotype, molecular, and cytogenetic phenotypes, can affect the outcome of chemotherapy and the rate of relapse, thus bringing great challenges to clinical treatment. AML patients exhibit multiple gene mutations, such as the nucleophosmin (NPM1) gene, the FMS-related tyrosine kinase 3 (FLT3) gene, the CCAAT/enhancer-binding protein alpha gene, and the myeloid-lymphoid or mixed-lineage leukemia gene. The mutation frequency of the NPM1 gene was the highest and is the only detectable genetic change in about $28 \%$ of cases. $^{38,39}$

In the recently updated WHO classification of acute leukemia, exon 12 mutations of the NPM1 gene have been defined as a distinct molecular leukemia entity. ${ }^{40,41}$ In this study, we confirmed that IL-37 expression was associated with NPM1 mutation in newly diagnosed AML patients. Therefore, our results demonstrate that IL-37 may be a predictive molecular marker of favorable prognosis of AML patients.

Recent studies indicate that dysregulated cytokine expression, which is a general hallmark of chronic inflammation and auto-inflammatory diseases, may also contribute to the development of hematological malignancies. ${ }^{23}$ We demonstrated that the levels of IL-6, described as key pro-inflammatory cytokines that promote inflammatory diseases and are involved in the regulation of hematopoiesis, were upregulated in newly diagnosed AML patients (Figure 5C). Van Loo et al found increased cytokine release of IL-6, IL-8, IL-10, and TNF $\alpha$ in whole blood 
A

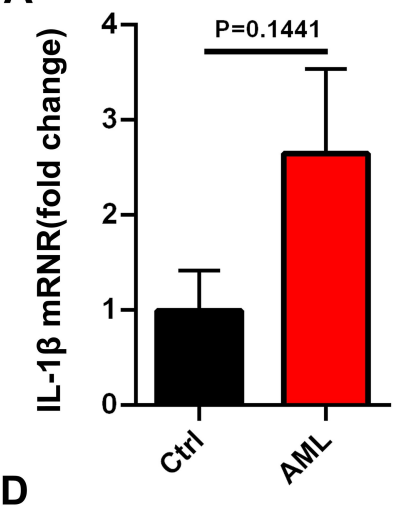

D

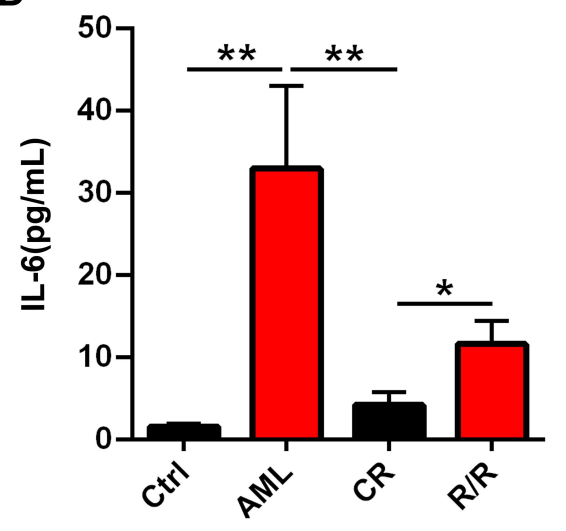

B

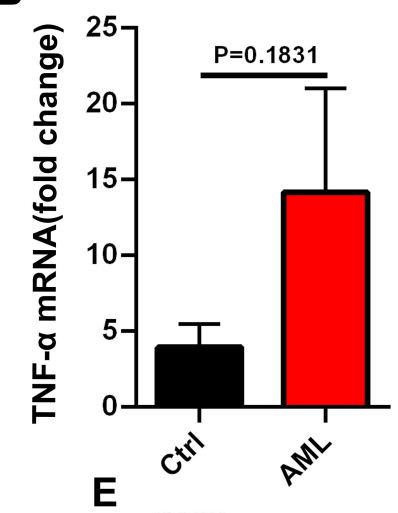

E

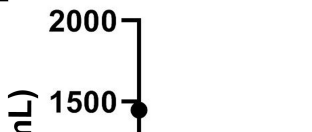

C

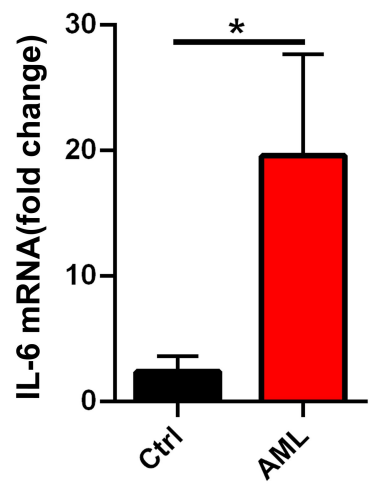

$r=-0.3682$

$p=0.0092$

Figure 5 IL-37 expression was associated with IL-6 in AML patients. (A-C) mRNA levels of IL-I $\beta$, TNF $\alpha$ and IL-6 in patients with newly diagnosed AML by qRT-PCR. (D) Serum IL-6 levels in patients with newly diagnosed AML $(n=21)$, CR AML $(n=20), R / R$ AML $(n=9)$ and healthy controls $(n=20)$ by ELISA. (E) Spearman correlation analysis showed a negative correlation of IL-37 with IL-6. Data show the mean \pm SD. **p $<0.01$, * $p<0.05$.

A

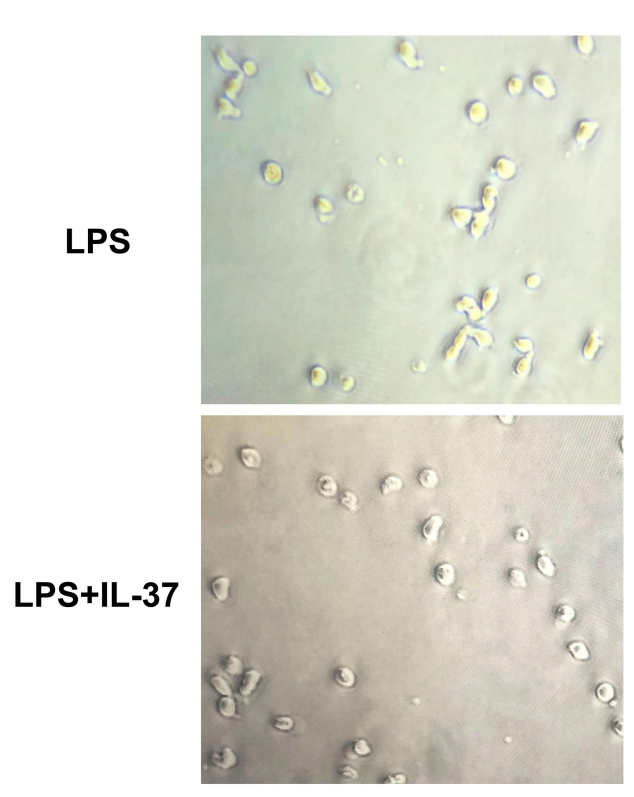

B

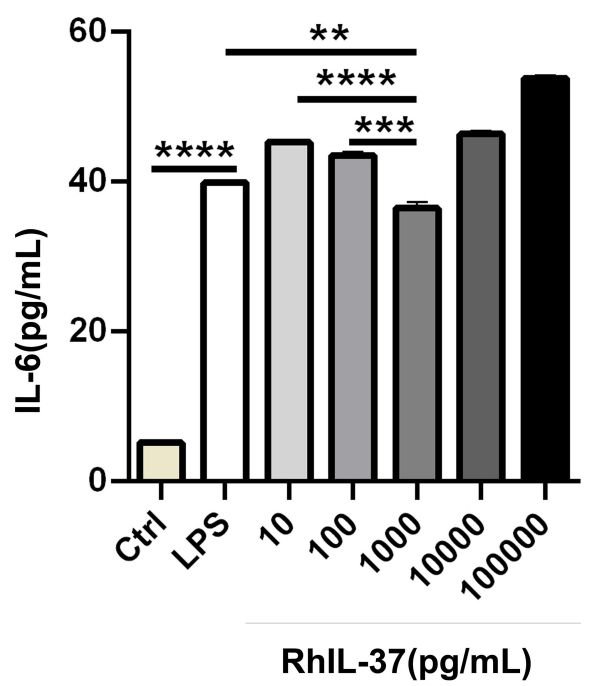

Figure 6 Human recombinant IL-37 inhibited IL-6 production of PBMC stimulated by LPS in AML patients. (A) Morphological changes of PBMC stimulated by LPS or IL-37 and LPS. (B) IL- 6 expression was detected by ELISA in the supernatant of PBMC stimulated by LPS or LPS and gradient concentration of rhIL-37. Data show the mean \pm SD. $* * * * \mathrm{P}<0.0001$, $* * * \mathrm{P}<0.001$ and $* * \mathrm{P}<0.01$. 
of AML patients in $2015 .^{42}$ In 2020 , Hou et al found that AML cells could remodel HS-5 cells to secrete more IL$6{ }^{43}$ These results are consistent with those of our study.

Interestingly, we found that IL-37 expression was negatively correlated with IL-6 expression in the serum (Figure $5 \mathrm{E})$. Reports indicate that IL-6 could stimulate the proliferation of blast cells and influence the prognosis of AML patients. $^{44,45}$ We next explored whether IL-37 could regulate the production of IL-6 in AML patients. Previous studies suggested that elevated levels of TLRs were observed in various hematological malignant cells, including primary AML cells, and associated with chemoresistance and monocytic differentiation. ${ }^{46,47}$ The pathogenand danger-associated molecular patterns such as teichoic acid, LPS, and lipoprotein could bind to these TLRs, recruit adaptor proteins, and initiate the signaling cascades subsequently, leading to the transcription of pro-inflammatory cytokines. $^{48-50}$ Therefore, we extracted PBMC, which contained many leukemic blasts from the peripheral blood of newly diagnosed AML patients, and added LPS and gradient concentrations of rhIL-37 into the supernatants. We found that the overexpressed IL-6 stimulated by LPS was markedly inhibited by rhIL-37 at $1 \mathrm{ng} / \mathrm{mL}$ (Figure 6B). Our results suggest that IL-37 may involved in the development of AML via the IL-6 signaling pathway.

In conclusion, our results showed that IL-37 is a potential indicator for the prognosis of AML patients. Moreover, IL-37 might participate in the pathophysiology of AML progression through regulating the IL-6 signaling pathway. Therefore, IL-37 offers new insights into the pathogenesis and potential therapeutic strategies.

\section{Acknowledgment}

Xiaonan Wei and Yulan Li share first authorship.

\section{Disclosure}

The authors report no conflicts of interest in this work.

\section{References}

1. Dohner H, Weisdorf DJ, Bloomfield CD. Acute Myeloid Leukemia. $N$ Engl J Med. 2015;373(12):1136-1152. doi:10.1056/NEJMra140 6184

2. Arber DA, Orazi A, Hasserjian R, et al. The 2016 revision to the World Health Organization classification of myeloid neoplasms and acute leukemia. Blood. 2016;127(20):2391-2405. doi:10.1182/blood2016-03-643544

3. Ishikawa F, Yoshida S, Saito Y, et al. Chemotherapy-resistant human AML stem cells home to and engraft within the bone-marrow endosteal region. Nat Biotechnol. 2007;25(11):1315-1321. doi:10.1038/ nbt1350
4. Ho TC, LaMere M, Stevens BM, et al. Evolution of acute myelogenous leukemia stem cell properties after treatment and progression. Blood. 2016;128(13):1671-1678. doi:10.1182/blood-2016-02-695312

5. Smith DE, Renshaw BR, Ketchem RR, Kubin M, Garka KE, Sims JE. Four new members expand the interleukin-1 superfamily. J Biol Chem. 2000;275(2):1169-1175. doi:10.1074/jbc.275.2.1169

6. Kumar S, McDonnell PC, Lehr R, et al. Identification and initial characterization of four novel members of the interleukin-1 family. $J$ Biol Chem. 2000;275(14):10308-10314. doi:10.1074/jbc.275.14.10308

7. Nold MF, Nold-Petry CA, Zepp JA, Palmer BE, Bufler P, Dinarello CA. IL-37 is a fundamental inhibitor of innate immunity. Nat Immunol. 2010;11(11):1014-1022. doi:10.1038/ni.1944

8. Tete S, Tripodi D, Rosati M, et al. IL-37 (IL-1F7) the newest anti-inflammatory cytokine which suppresses immune responses and inflammation. Int J Immunopathol Pharmacol. 2012;25(1):31-38. doi:10.1177/039463201202500105

9. Taylor SL, Renshaw BR, Garka KE, Smith DE, Sims JE. Genomic organization of the interleukin-1 locus. Genomics. 2002;79 (5):726-733. doi:10.1006/geno.2002.6752

10. Boraschi D, Lucchesi D, Hainzl S, et al. IL-37: a new anti-inflammatory cytokine of the IL-1 family. Eur Cytokine Netw. 2011;22(3):127-147. doi:10.1684/ecn.2011.0288

11. Chen H-M, Fujita M. IL-37: a new player in immune tolerance. Cytokine. 2015;72(1):113-114. doi:10.1016/j.cyto.2014.11.025

12. Dinarello CA, Nold-Petry C, Nold M, et al. Suppression of innate inflammation and immunity by interleukin-37. Eur $J$ Immunol. 2016;46(5):1067-1081. doi:10.1002/eji.201545828

13. McNamee EN, Masterson JC, Jedlicka P, et al. Interleukin 37 expression protects mice from colitis. Proc Natl Acad Sci U S A. 2011;108 (40):16711-16716. doi:10.1073/pnas.1111982108

14. Ballak DB, van Diepen JA, Moschen AR, et al. IL-37 protects against obesity-induced inflammation and insulin resistance. Nat Commun. 2014;5:4711. doi:10.1038/ncomms5711

15. Ye L, Jiang B, Deng J, et al. IL-37 alleviates rheumatoid arthritis by suppressing IL-17 and IL-17-triggering cytokine production and limiting Th17 cell proliferation. J Immunol. 2015;194(11):5110-5119. doi:10.4049/jimmunol.1401810

16. Ye L, Ji L, Wen Z, et al. IL-37 inhibits the production of inflammatory cytokines in peripheral blood mononuclear cells of patients with systemic lupus erythematosus: its correlation with disease activity. J Transl Med. 2014;12:69. doi:10.1186/1479-5876-12-69

17. Bulau AM, Fink M, Maucksch $C$, et al. In vivo expression of interleukin-37 reduces local and systemic inflammation in concanavalin A-induced hepatitis. Sci World J. 2011;11:2480-2490. doi:10.1100/2011/968479

18. Teng $\mathrm{X}, \mathrm{Hu} \mathrm{Z}$, Wei $\mathrm{X}$, et al. IL-37 ameliorates the inflammatory process in psoriasis by suppressing proinflammatory cytokine production. $J$ Immunol. 2014;192(4):1815-1823. doi:10.4049/ jimmunol.1300047

19. Li Y, Zhao M, Guo C, et al. Intracellular mature IL-37 suppresses tumor metastasis via inhibiting Rac1 activation. Oncogene. 2018;37 (8):1095-1106. doi:10.1038/onc.2017.405

20. Wang S, An W, Yao Y, et al. Interleukin 37 expression inhibits STAT3 to suppress the proliferation and invasion of human cervical cancer cells. $J$ Cancer. 2015;6(10):962-969. doi:10.7150/jca.12266

21. Zhao JJ, Pan QZ, Pan K, et al. Interleukin-37 mediates the antitumor activity in hepatocellular carcinoma: role for CD57+ NK cells. Sci Rep. 2014;4:5177. doi:10.1038/srep05177

22. Li ZC, Sun MD, Zheng YQ, Fu HJ. The low expression of IL-37 involved in multiple myeloma - associated angiogenesis. Med Sci Monit. 2016;22:4164-4168. doi:10.12659/MSM.897451

23. Kristinsson SY, Bjorkholm M, Hultcrantz M, Derolf AR, Landgren O, Goldin LR. Chronic immune stimulation might act as a trigger for the development of acute myeloid leukemia or myelodysplastic syndromes. J Clin Oncol. 2011;29(21):2897-2903. doi:10.1200/JCO.2011.34.8540 
24. Ramadan SM, Fouad TM, Summa V, Hasan S, Lo-Coco F. Acute myeloid leukemia developing in patients with autoimmune diseases. Haematologica. 2012;97(6):805-817. doi:10.3324/haematol.2011. 056283

25. Binder S, Luciano M, Horejs-Hoeck J. The cytokine network in acute myeloid leukemia (AML): a focus on pro- and anti-inflammatory mediators. Cytokine Growth Factor Rev. 2018;43:8-15. doi:10.10 16/j.cytogfr.2018.08.004

26. Brazvan B, Farahzadi R, Mohammadi S, et al. Key immune cell cytokines affects the telomere activity of cord blood cells in vitro. Adv Pharm Bull. 2016;6:153-161. doi:10.15171/apb.2016.022

27. Verma G, Raigond B, Pathania S, Kochhar T, Naga K. Development and comparison of reverse transcription-loop-mediated isothermal amplification assay (RT-LAMP), RT-PCR and real time PCR for detection of Potato spindle tuber viroid in potato. Eur J Plant Pathol. 2020;158:951-964. doi:10.1007/s10658-020-02129-z

28. Huh H, Park J, Kim J, et al. Performance of the Real-Q EBV quantification kit for epstein-barr virus DNA quantification in whole blood. Ann Lab Med. 2017;37:147-150. doi:10.3343/alm.2017.37.2.147

29. Sun $X, G e X, X u Z$, Chen D. Identification of circular RNAmicroRNA-messenger RNA regulatory network in hepatocellular carcinoma by integrated analysis. $J$ Gastroenterol Hepatol. 2019;35:157-164. doi:10.1111/jgh.14762

30. Fathi E, Valipour B, Sanaat Z, Nozad Charoudeh H, Farahzadi R. Interleukin- $6,-8$, and TGF- $\beta$ secreted from mesenchymal stem cells show functional role in reduction of telomerase activity of leukemia cell via Wnt5a/ $\beta-C a t e n i n$ and P53 pathways. Adv Pharm Bull. 2020;10:307-314. doi:10.34172/apb.2020.037

31. Farahzadi R, Fathi E, Vietor I. Mesenchymal stem cells could be considered as a candidate for further studies in cell-based therapy of alzheimer's disease via targeting the signaling pathways. ACS Chem Neurosci. 2020;11:1424-1435. doi:10.1021/acschemneuro.0c00052

32. Abulkhir A, Samarani S, Amre D, et al. A protective role of IL-37 in cancer: a new hope for cancer patients. J Leukoc Biol. 2017;101 (2):395-406. doi:10.1189/jlb.5RU0816-341R

33. Quirk S, Agrawal DK. Immunobiology of IL-37: mechanism of action and clinical perspectives. Expert Rev Clin Immunol. 2014;10 (12):1703-1709. doi:10.1586/1744666X.2014.971014

34. Bufler P, Gamboni-Robertson F, Azam T, Kim SH, Dinarello CA. Interleukin-1 homologues IL-1F7b and IL-18 contain functional mRNA instability elements within the coding region responsive to lipopolysaccharide. Biochem J. 2004;381(Pt 2):503-510. doi:10.1042/ BJ20040217

35. Carey A, Edwards D, Eide CA, et al. Identification of interleukin-1 by functional screening as a key mediator of cellular expansion and disease progression in acute myeloid leukemia. Cell Rep. 2017;18 (13):3204-3218. doi:10.1016/j.celrep.2017.03.018

36. Jia H, Liu J, Han B. Reviews of interleukin-37: functions, receptors, and roles in diseases. BioMed Res Int. 2018;2018:1-14.
37. Zhao M, Li Y, Guo C, et al. IL-37 isoform D downregulates pro-inflammatory cytokines expression in a Smad3-dependent manner. Cell Death Dis. 2018;9(6):582. doi:10.1038/s41419-018-0664-0

38. Pourrajab F, Zare-Khormizi MR, Hashemi AS, Hekmatimoghaddam S. Genetic characterization and risk stratification of acute myeloid leukemia. Cancer Manag Res. 2020;12:2231. doi:10.2147/CMAR. S242479

39. Dohner K, Döhner H. Molecular characterization of acute myeloid leukemia. Haematologica. 2008;93(7):976-982. doi:10.3324/ haematol.13345

40. Shipley JL, Butera JN. Acute myelogenous leukemia. Exp Hematol. 2009;37(6):649-658. doi:10.1016/j.exphem.2009.04.002

41. Martelli MP, Sportoletti P, Tiacci E, Martelli MF, Falini B. Mutational landscape of AML with normal cytogenetics: biological and clinical implications. Blood Rev. 2013;27(1):13-22. doi:10.1016/ j.blre.2012.11.001

42. Van Loo P, Doornbos R, Dolstra H, Shamsili S, Bakker L. Preclinical evaluation of MCLA117, a CLEC12AxCD3 bispecific antibody efficiently targeting a novel leukemic stem cell associated antigen in AML. Blood. 2015;126:325. doi:10.1182/blood.V126.23.325.325

43. Hou D, Wang B, You R, et al. Stromal cells promote chemoresistance of acute myeloid leukemia cells via activation of the IL-6/STAT3/ OXPHOS axis. Ann Transl Med. 2020;8:1346. doi:10.21037/atm-203191

44. Ikebuchi K, Wong GG, Clark SC, Ihle JN, Hirai Y, Ogawa M. Interleukin 6 enhancement of interleukin 3-dependent proliferation of multipotential hemopoietic progenitors. Proc Natl Acad Sci U S A. 1987;84(24):9035-9039. doi:10.1073/pnas.84.24.9035

45. Oster W, Cicco NA, Klein H, et al. Participation of the cytokines interleukin 6, tumor necrosis factor-alpha, and interleukin 1-beta secreted by acute myelogenous leukemia blasts in autocrine and paracrine leukemia growth control. J Clin Invest. 1989;84 (2):451-457. doi:10.1172/JCI114186

46. Monlish DA, Bhatt ST, Schuettpelz LG. The role of toll-like receptors in hematopoietic malignancies. Front Immunol. 2016;7:390. doi:10.3389/fimmu.2016.00390

47. Rybka J, Butrym A, Wrobel T, et al. The expression of Toll-like receptors in patients with acute myeloid leukemia treated with induction chemotherapy. Leuk Res. 2015;39(3):318-322. doi:10.1016/j. leukres.2015.01.002

48. Wang X, Smith C, Yin H. Targeting Toll-like receptors with small molecule agents. Chem Soc Rev. 2013;42(12):4859-4866. doi:10.1039/c3es60039d

49. De Nardo D. Toll-like receptors: activation, signalling and transcriptional modulation. Cytokine. 2015;74(2):181-189. doi:10.1016/j. cyto.2015.02.025

50. Kawai T, Akira S. The role of pattern-recognition receptors in innate immunity: update on Toll-like receptors. Nat Immunol. 2010;11 (5):373-384. doi:10.1038/ni.1863

\section{Publish your work in this journal}

Cancer Management and Research is an international, peer-reviewed open access journal focusing on cancer research and the optimal use of preventative and integrated treatment interventions to achieve improved outcomes, enhanced survival and quality of life for the cancer patient.
The manuscript management system is completely online and includes a very quick and fair peer-review system, which is all easy to use. Visit http://www.dovepress.com/testimonials.php to read real quotes from published authors. 